\title{
Occipital and orbitofrontal hemodynamics during naturally paced reading: An fNIRS study
}

\author{
Markus J. Hofmann a,b,c,*, Michael Dambacher ${ }^{\text {d }}$, Arthur M. Jacobs ${ }^{\text {b,c }}$, Reinhold Kliegl ${ }^{\text {d }}$, Ralph Radach ${ }^{\text {a }}$, \\ Lars Kuchinke $^{\mathrm{e}}$, Michael M. Plichta ${ }^{\mathrm{f}}$, Andreas J. Fallgatter ${ }^{\mathrm{g}, \mathrm{h}, \mathrm{i}}$, Martin J. Herrmann ${ }^{\mathrm{i}}$ \\ a Department of Psychology, University of Wuppertal, Max-Horkheimer Str. 20, 42119 Wuppertal, Germany \\ b Department of Psychology, Habelschwerdter Allee 45, 14195 Berlin, Germany \\ c Dahlem Institute for Neuroimaging of Emotion (D.I.N.E.), Free University Berlin, Germany \\ d Department of Psychology, University Potsdam, Karl-Liebknecht-Str. 24/25, 14476 Potsdam, Germany \\ e Department of Psychology, Ruhr-University Bochum, Universitaetsstr. 150, 44780 Bochum, Germany \\ ${ }^{\mathrm{f}}$ Central Institute of Mental Health, Department of Psychiatry and Psychotherapy, Medical Faculty Mannheim, Heidelberg University, J5, 68159 Mannheim, Germany \\ ${ }^{\mathrm{g}}$ Department of Psychiatry and Psychotherapy, University of Tuebingen, Calwerstr. 14, 72076 Tuebingen, Germany \\ h LEAD Graduate School, University of Tuebingen, Calwerstr. 14, 72076 Tuebingen, Germany \\ ${ }^{i}$ Department for Psychiatry, Psychosomatics and Psychotherapy, University Wuerzburg, Fuechsleinstr. 15, 97080 Wuerzburg, Germany
}

\begin{abstract}
A B S T R A C T
Humans typically read at incredibly fast rates, because they predict likely occurring words from a given context. Here, we used functional near infrared spectroscopy (fNIRS) to track the ultra rapid hemodynamic responses of words presented every $280 \mathrm{~ms}$ in a naturally paced sentence context. We found a lower occipital deoxygenation to unpredictable than to predictable words. The greater hemodynamic responses to unexpected words suggest that the visual features of expected words have been pre activated previous to stimulus presentation. Second, we tested opposing theoretical proposals about the role of the medial orbitofrontal cortex (OFC): Either OFC may respond to the breach of expectation; or OFC is activated when the present stimulus matches the prediction. A significant interaction between word frequency and predictability indicated OFC responses to breaches of ex pectation for low but not for high frequency words: OFC is sensitive to both, bottom up processing as mediated by word frequency, as well as top down predictions. Particularly, when a rare word is unpredictable, OFC be comes active. Finally, we discuss how a high temporal resolution can help future studies to disentangle the hemo dynamic responses of single trials in such an ultra rapid event succession as naturally paced reading.
\end{abstract}

\section{Introduction}

The cognitive system uses context information to predict upcoming stimuli (Bar et al., 2006; Friston, 2010; Rao and Ballard, 1999; Rauss et al., 2011; Summerfield and Egner, 2009). When a stimulus is presented, it can either match these predictions or not. For instance, a sentence like "The road to hell is paved with good inventions" ends with a prediction error. Most readers would predict this sentence to end with 'intentions', as expected by the famous proverb.

The present study targets the function of two different brain regions in making predictions. On the one hand, Rao and Ballard (1999) suggest

\footnotetext{
* Corresponding author at: Room Z.01.11, Max-Horkheimer Str. 20, 42119 Wuppertal, Germany.

E-mail addresses: mhofmann@uni-wuppertal.de (M.J. Hofmann), michael.dambacher@uni-konstanz.de (M. Dambacher), ajacobs@zedat.fu-berlin.de (A.M. Jacobs), kliegl@uni-potsdam.de (R. Kliegl), radach@uni-wuppertal.de (R. Radach), lars.kuchinke@rub.de (L. Kuchinke), michael.plichta@zi-mannheim.de (M.M. Plichta), Andreas.Fallgatter@med.uni-tuebingen.de (A.J. Fallgatter). Herrmann_m@klinik.uni-wuerzburg.de (M.J. Herrmann).
}

that higher level visual areas generate predictions of lower level neural activation in the occipital cortex. On the other hand, the medial orbitofrontal cortex (OFC) has been suggested to host the generation of expectations (Bar et al., 2006; Nobre et al., 1999).

When an unexpected word like 'inventions' occurs during natural reading, word recognition takes more time and a second look at this word becomes likely (Rayner, 1998). If a second look is impossible because of sequential stimulus presentation, two recent studies show that unpredictable visual events lead to greater activation in the occip ital gyrus (Alink et al., 2010; Fischer et al., 2012; cf. Obleser and Kotz, 2010). Such effects are explainable by a predictive coding framework, which suggests that visual representations of expected events can be come active previous to stimulus exposure (Rao and Ballard, 1999; Rauss et al., 2011). Similarly, the free energy principle suggests that an iterative search for a plausible 'cause' for the unexpected event can explain such a greater occipital activation (Friston, 2010). As long as higher level information mismatches lower level information, predic tion errors occur. To test whether predictive coding within the free energy principle can also account for primary visual processes during 
reading, we manipulated word predictability. For this purpose, the results of a pre experimental cloze task were used. High and low pre dictability words were defined by their respective cloze completion probability (Dambacher et al., 2009).

A critical target region engaged in the generation of such visual expectations is the medial orbitofrontal cortex (OFC). In contrast to the generally accepted role of the occipital cortex in primary visual pro cesses, the role of the OFC is elusive. There are controversial findings how this region responds to stimuli that either match or violate predic tions. Nobre et al. (1999) proposed that the breach of expectation engages the OFC (Petrides, 2007; Schoenbaum et al., 2009). If a cueing stimulus predicts the spatial or temporal occurrence of a target stimu lus, unexpected events elicit OFC activation (Nobre et al., 1999). The OFC's role in violated predictions was often demonstrated using rewarding stimuli (e.g. Berns et al., 2001; Kringelbach and Rolls, 2004; O'Doherty et al., 2003; Petrides, 2007; Wallis, 2007). For instance, Berns et al. (2001) showed that the OFC responds stronger to mildly pleasurable stimuli such as fruit juice when they are unpredictable.

In contrast to Nobre et al.'s (1999) proposal, Bar et al. (2006) suggest that the OFC becomes active when top down predictions match rapidly incoming bottom up information. Therefore, correctly recognized pictures lead to greater OFC activation than incorrect recognition. More over, the authors manipulate the spatial frequency of pictures to vary the amount of available bottom up information. They suggest that low spatial frequency information is submitted fast to the OFC, which in turn generates top down predictions. These predictions are validated against high spatial frequency information, which becomes available later. When bottom up and top down informations match, OFC becomes active at around $130 \mathrm{~ms}$ after stimulus exposure (Bar et al., 2006).

Although both of the complementary theoretical proposals OFC responding either to prediction error or to match appear to contradict each other, they agree that timing is crucial (e.g., Kiebel et al., 2008). When stimulus presentation is too long in a recognition task, there is no need to rely on top down predictions and OFC activation diminishes. In this case, bottom up information is sufficient to recognize the stimu lus (Bar et al., 2006).

In visual word recognition, there is an elegant way to manipulate the speed with which bottom up information is submitted to higher level processes. Access to a hypothetical mental lexicon is faster to frequent than to rare words (Grainger and Jacobs, 1996; McClelland and Rumelhart, 1981). Therefore, we manipulated the frequency with which a word occurs in the natural language in addition to word predictability.

Word frequency effects are relatively well understood. They can be explained by Hebbian learning: Repeated exposure strengthens the connections of the neurons representing a word (e.g., Hauk and Pulvermüller, 2004; Hebb, 1949; Seidenberg and McClelland, 1989). Therefore, high frequency words are processed with increased synaptic efficacy (Pulvermüller, 1999). In event related potentials (ERP), this leads to lower amplitudes to high frequency words within $200 \mathrm{~ms}$ after stimulus exposure, which provides an upper time limit for the access to the mental lexicon (Dambacher et al., 2006; Hauk and Pulvermüller, 2004; Sereno et al., 1998). State of the art models of word recognition suggest that high frequency word recognition is based on a fast visual orthographic match, while particularly low frequency word rec ognition profits from processing after $250 \mathrm{~ms}$. In this late period, for instance, phonological information contributes to word recognition (Coltheart et al., 2001; Grainger and Holcomb, 2009; Perry et al., 2007; Proverbio et al., 2004). Larger predictability effects for low frequency words in this time frame support this (Dambacher et al., 2006; Kutas and Federmeier, 2011). During natural reading, single word fixations take around $250 \mathrm{~ms}$ (Rayner, 1998), but particularly low frequency word processing is still underway when the eyes move to the next word (Kliegl et al., 2006).

When aiming to investigate occipital and orbitofrontal hemody namic responses in a naturally paced sentence context, however, natural reading rates of around three to five words per second pose a problem for the relatively low temporal resolution of fMRI tech niques with a typical sampling rate of about $0.5 \mathrm{~Hz}$. Several solutions were proposed to circumvent this limitation while addressing lan guage processing.

The first and most commonly used solution is to present subjects with whole sentences. Though the experimental manipulation in this case is often at the word level, local blood oxygenation is modeled as a response to sentence presentation (e.g., Bohrn et al., 2012). Thus, it is confounded by the hemodynamic responses of the non target words. A second solution is to present the words at slower than natural pre sentation rates (e.g., Fiebach et al., 2004). This, however, is problematic for the investigation of sentence processing, because slow presentation rates lead to unnatural processing strategies. For instance, an early interaction between predictability and word frequency occurs only at natural, but not at artificially slow reading rates (Dambacher et al., 2012). This is in line with Bar et al.'s (2006) proposal that slow presen tation rates engage a largely bottom up driven processing strategy, which prevents the OFC from becoming active. Third, as several ultra rapid hemodynamic responses sum up in an approximately linear fashion (Burock et al., 1998; but cf. Boynton et al., 1996), Yarkoni et al. (2008) propose to add the predictor variables of many words to account for hemodynamic responses. A fourth option is to trade off a bit of fMRI's outstanding spatial resolution for sampling frequency (e.g., Sabatinelli et al., 2009). Even with a typical sampling frequency, however, the investigation of the hemodynamic response in the OFC leads to large drop off rates (e.g., Kringelbach and Rolls, 2004). Thus specialized stimulation sequences were developed for this region (e.g., Schmidt et al., 2005).

Here we pioneer an alternative approach. Functional near infrared spectroscopy (fNIRS) allows a sufficient sampling frequency for tracking ultra rapid hemodynamic responses. This method is a reliable tool to investigate visual word recognition (Chen et al., 2011; Hofmann et al., 2008; Noguchi et al., 2002; Plichta et al., 2006), and can sensitively detect deoxyhemoglobin changes in the OFC (Brink et al., 2011). Neural activation is indicated by a decrease of deoxygenated hemoglobin, which indexes neural energy consumption (Buxton et al., 2004; Mandeville et al., 1999; Raichle and Mintun, 2006). Therefore, we used optical imag ing to investigate the hemodynamic response, when word recognition is as fast as in natural reading (Devor et al., 2012).

\section{Material and methods}

\section{Participants}

28 right handed native German speakers participated in the present study ( 21 females, mean age: $25.27, \mathrm{SE}=1.34$ ). They had corrected or corrected to normal sight, reported no history of neurological or lan guage disorders, and received $12 €$ for participation. All procedures were in accordance with the 2008 Declaration of Helsinki. Participants gave written informed consent after full explanation of procedures.

\section{Materials}

Stimuli were taken from Dambacher et al. (2009, 2012). Each partic ipant read 144 sentence units. Respectively 36 sentence units reflected one experimental condition in the $2 \times 2$ experimental design of the factors word frequency (low/high) and predictability (low/high). Each sentence unit is composed of two context sentences and one neutral sentence (cf. Fig. 1 for example). In the neutral sentences, a target word of low frequency was presented to half of the participants, while the other half were exposed to a high frequency word. Low frequency words occurred rarer than 10 times per million of written text, while high frequency words occurred at least 100 times per million words (Geyken, 2007). Length and target position in the sentences were care fully matched. 

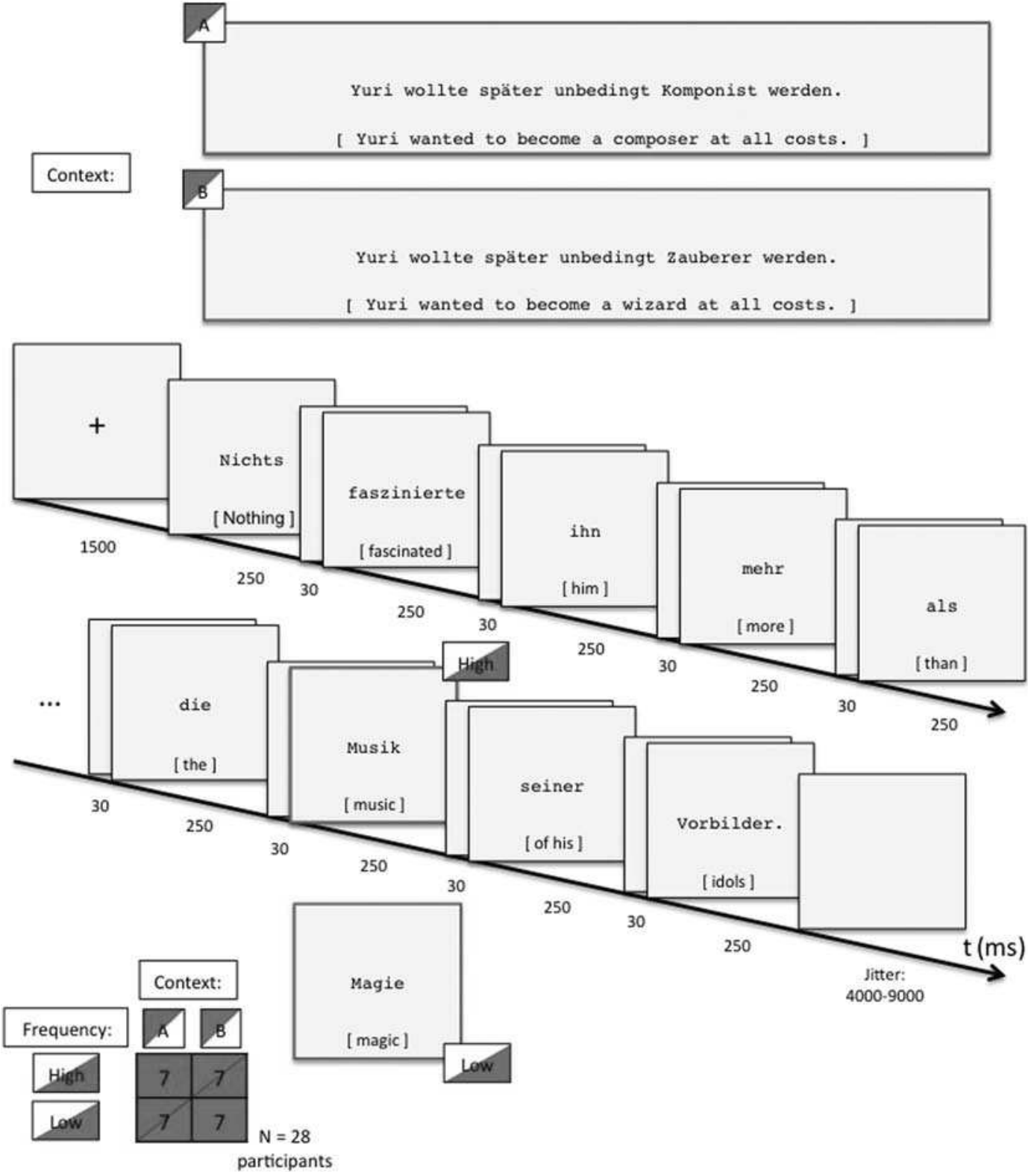

Fig. 1. Experimental design and procedure are exemplified on one sentence unit. Half of the participants were exposed to context sentence A (upper-red), and the other half to context B (upper-green). Context sentence presentation was terminated by a button press. The fixation cross was exposed for $1500 \mathrm{~ms}$. Then a neutral sentence frame was shown word-by-word. Words were presented for $250 \mathrm{~ms}$ and followed by a $30 \mathrm{~ms}$ blank screen. Target stimuli were embedded into this neutral sentence. They were of either high (lower-red) or low frequency (lower-green). Each participant saw 36 of the 144 sentence units in one of the conditions. Sentence conditions were balanced across participants according to Latin Square, such that one sentence unit was presented a quarter of the participants in one condition, respectively ( $\mathrm{N}=7$ of a total of 28 participants). Congruent colors code for high predictability, while incongruent colors index low predictability.

High predictability of a target word was induced by a prior context sentence that triggered the expectancy of either the high or the low frequency target word. Predictability norms of targets were assessed from 151 subjects in an independent cloze study. High predictability was defined by a cloze completion probability greater than 0.5 (mean $=.8$ ), and low predictability by a probability lower than 0.1 (mean $=.01)$. None of the cloze test subjects participated in the present experiment (see Dambacher et al., 2012, for further details about the material).

Each participant saw only one version of a sentence unit, that is, target word frequency and predictability were counterbalanced across subjects. For a given target word, low and high predictability condi tions differ only in the preceding context sentence. Thus, effects of predictability were measured on physically identical target words em bedded in the same neutral sentences (cf. Fig. 1).

\section{Procedure}

Stimuli were exposed on a $17 \mathrm{in.} \mathrm{monitor} \mathrm{(} 70 \mathrm{~Hz}$ screen refresh rate) using Presentation Software on a Windows XP computer. Cerebral oxygenation changes were recorded by a Hitachi optical topograph (ETG 4000, Hitachi Medical Co., Kashiwa, Japan), which allowed for a sampling frequency of $10 \mathrm{~Hz}$. This continuous wave device measures changes in light attenuation at 2 wavelengths (695 and $830 \mathrm{~nm}, \pm 20$ $\mathrm{nm}$ ) and hence allows for the differentiation of two dynamic absorbers (oxygenated and deoxygenated hemoglobin). Concentration changes of [deoxy $\mathrm{Hb}$ ] are obtained from a modified Beer Lambert approach (Cope and Delpy, 1988). The probe set contained 16 light emitters and 17 detectors, which results in 52 channels. These optodes were attached on a plastic shell with an inter optode distance of $3 \mathrm{~cm}$. Using this array, we covered an area of $\sim 6 \times 30 \mathrm{~cm}$. The probe array was positioned on 
the subject's head with the medial detector of the lowest optode row corresponding to T3 of the 1020 system. The lower edge of the probe set was defined to be $1 \mathrm{~cm}$ above the nasion. In sum, the present probeset definition allowed for investigating the largest part of the left hemisphere, as well as the frontal and occipital poles (cf. Fig. 2 below; Hofmann et al., 2008).

After eight practice trials, subjects read 144 experimental sentence pairs. There was also a short break after half of the experimental trials, terminated by the subject's keypress. All words were presented in black letters on a light gray screen in courier font (size 18). Each trial started with the presentation of a context sentence that was entirely displayed on the screen. Participants pressed a button after they had read and understood the context sentences. Following a blank screen of $500 \mathrm{~ms}$, a fixation cross was presented for $1000 \mathrm{~ms}$, followed by another blank screen of $500 \mathrm{~ms}$. Then, the neutral sentence (including the target word) was presented word by word at the screen center. Each word was displayed for $250 \mathrm{~ms}$ and followed by a blank screen of $30 \mathrm{~ms}$. Following the last word of the neutral sentence, presentation of a blank screen randomly varied between 4500 and $12,500 \mathrm{~ms}$. After another blank of $500 \mathrm{~ms}$, either the next trial started ( $67 \%$ of the trials), or a three alternative multiple choice question (33\% of the trials) tested sentence comprehension. An overall error percentage of $1.49 \%$ suggests that the participants generally read and understood the sentences.

\section{Data analysis}

Event related oxygenation changes were analyzed by a 2 staged GLM (Plichta et al., 2006; Schroeter et al., 2004). As oxygenated hemo globin is prone to be contaminated by extracerebral artifacts due to skin oxygenation in the OFC (Haeussinger et al., 2011; Kirilina et al., 2012), the present study focused on deoxyhemoglobin, which is the major constituent of the BOLD response (Steinbrink et al., 2006). To correct for artifacts due to heartbeat, data were band pass filtered to include signals between 0.02 and $0.7 \mathrm{~Hz}$.

The first level analysis comprised 9 predictors. Four predictors coded the experimental conditions, that is, the $2 \times 2$ design with the experimental factors of frequency (low/high) and predictability (low/ high). Additional five predictors coded the fixation cross, the presenta tion of the context sentence, the question, a button press, as well as a single predictor for non target words in the neutral sentences. These five predictors were excluded from second level analyses. We generated the GLM predictors by convolving a Gaussian function with each event (duration $=15 \mathrm{~s} ; \mathrm{SD}=2.5 \mathrm{~s}$; Plichta et al., 2007). To absorb variance resulting from interindividual differences in the onset and the dispersion of the hemodynamic response, first and second derivatives of each prediction term were included. Beta values for each predictor were deter mined by optimizing the model to data fit by a least squared error procedure (Bullmore et al., 1996). Finally, we fitted a first order auto regressive model to the resulting residuals and removed serial autocorrelated error variance from the final model re estimation (Cochrane and Orcutt, 1949; Plichta et al., 2007).

In the second level analysis, the four first level beta values of the ex perimental conditions obtained for each subject were submitted to a $2 \times$ 2 repeated measures ANOVA. To correct for false positives due to alpha inflation, partial Bonferroni correction was applied (Sankoh et al., 1997). The Dubey/Armitage Parmar alpha boundary considers the mean intercorrelation of 0.41 between the channels. Therefore, the channels are not treated as fully independent samples. As the mean inter correlation of the channels was 0.41 , the Bonferroni corrected alpha of $\mathrm{P}<0.05$ (0.01) corresponds to an uncorrected significance threshold of $\mathrm{P}<0.0049$ (0.00097).

To obtain an estimate of the empirical hemodynamic responses of the target events, we purged the critical hemodynamic responses from the non target responses in a deconvolution analysis (Koch et al., 2009). For each of the four target conditions, we subtracted the eight remaining predictors from the empirical hemodynamic responses. Averages time locked to the event of the respective critical stimulus presentation were generated for each participant and experimental condition relative to the mean of a 1 second pre stimulus epoch. Finally, we averaged these time courses across participants for each experimen tal condition, and calculated the difference waves for low minus high predictability (see Figs. 6 and 7).

To allow for anatomic inference of the channel positions, we applied a virtual optode registration technique (Tsuzuki et al., 2007), which was documented in Hofmann et al. (2008). The general rational is to attach a virtual probe set by the same anatomic definition points on 1000 simulated heads. Standard deviations of registration accuracy are indicated by the size of the channel positions, and given in Table 1. $\mathrm{x} / \mathrm{y} / \mathrm{z}$ coordinates are given in MNI space, and anatomically labeled by Tzourio Mazoyer et al.'s (2002) brain atlas.

\section{Results}

The $2 \times 2$ repeated measures ANOVA at the second level revealed a significant main effect of predictability in an occipital channel (Fig. 2; Table 1). This channel has been termed OCC. Fig. 3 shows the beta value differences of low high predictability, separately for low and high frequency words. Planned comparisons revealed an effect of pre dictability for low $(\mathrm{t}(27)=-3.24, \mathrm{P}<0.01$, uncorrected $)$, and for high frequency words $(\mathrm{t}(27)=-2.01 ; \mathrm{P}<0.05)$, respectively.

Moreover, we obtained significant interactions between frequency and predictability in two channels located in the OFC (Fig. 4). These channels were termed OFC 1 and OFC 2 . The estimated beta value dif ferences can be examined in Fig. 5. While planned comparisons for

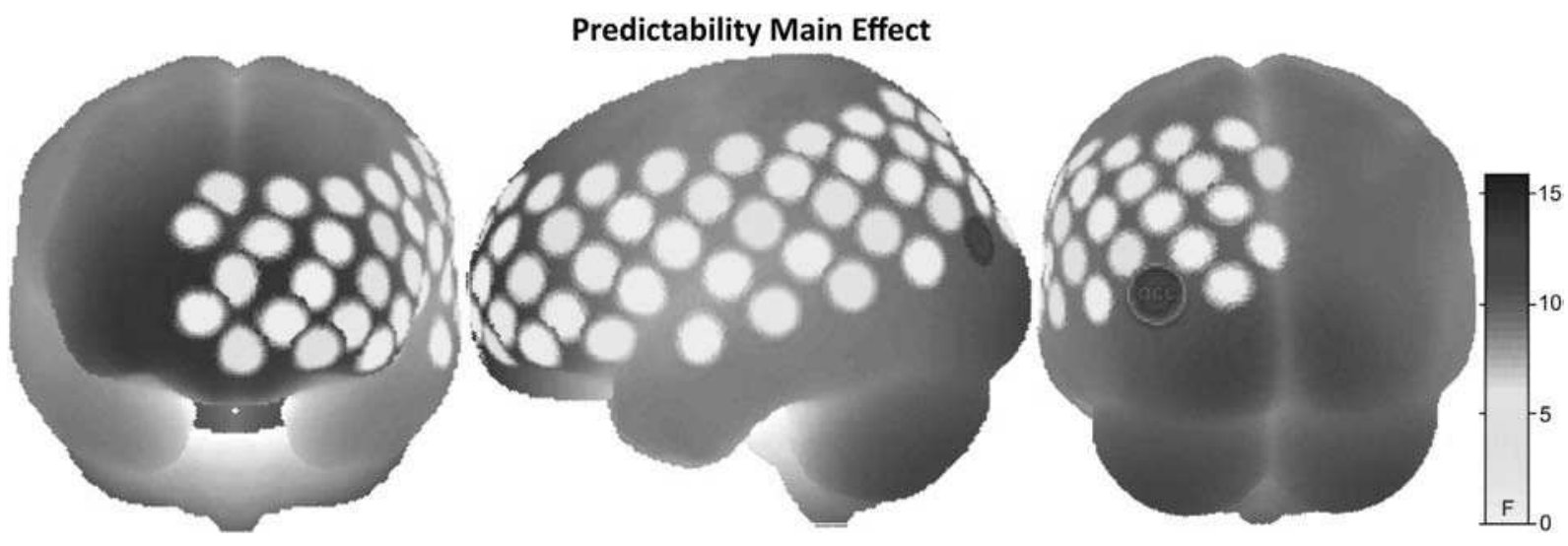

Fig. 2. The figure shows the results of the repeated measures ANOVAs for the main effect of predictability. Resulting F-values are color-coded. The only channel that surpassed a Bonferronicorrected threshold is hence termed OCC (cf. Fig. 3 and 6). 
Table 1

Results of the $2 \times 2$ rmANOVA. The first column indexes the effect, and the second column the channel label (cf. Figs. 2-5). We found one channel reaching the Bonferroni-corrected significance threshold for the main effect of predictability (Figs. 2 and 3). Two channels revealed a reliable interaction between word frequency and predictability (Figs. 4 and 5). The next columns show the most likely MNI-x/y/z coordinates and the localization due to Tzourio-Mazoyer et al.'s (2002) brain atlas. Anatomical standard deviation of the localization approach is given in brackets $(\mathrm{mm})$.

\begin{tabular}{|c|c|c|c|c|c|}
\hline Effect & Channel & Localization & $\begin{array}{l}\mathrm{x} / \mathrm{y} / \mathrm{z} \\
(\mathrm{SD})\end{array}$ & $\mathrm{F}$ & $\mathrm{P}$ \\
\hline Predictability & OCC & Occipital, middle, left & $37 / 91 / 20(12)$ & 11.7 & $<0.05$ \\
\hline \multirow[t]{2}{*}{ Frequency $\times$ predictability } & OFC-1 & Frontal, superior/medial, orbital, right & $13 / 71 / \quad 1(10)$ & 9.6 & $<0.05$ \\
\hline & OFC-2 & Frontal, medial, orbital, left & $1 / 68 / 13(9)$ & 15.5 & $<0.01$ \\
\hline
\end{tabular}

OFC 1 revealed only nonsignificant trends towards a predictability effects in low $(\mathrm{t}(27)=-1.51 ; \mathrm{P}=0.14)$, and in high frequency words $(t(27)=1.67 ; \mathrm{P}=0.09)$, the opposite signs of the $t$ values account for the interaction. For low frequency words, planned compar isons revealed a reliable deoxygenation decrease to low predictability in channel OFC $2(\mathrm{t}(27)=-2.14, \mathrm{P}<0.05)$.

\section{Discussion}

The present study demonstrated that humans can read at incredibly fast rates, because they have concrete hypotheses about which words are likely to occur in a given context. First, low predictability words elicited a [deoxy $\mathrm{Hb}$ ] decrease in the occipital gyrus relative to high predictability words. This results from a greater wash out of consumed oxygen (Buxton et al., 2004; Mandeville et al., 1999), which suggests that neural activation is greater to low predictability words. The effect can be explained by the top down activation of visual features of expected words prior to target presentation. Second, predictability interacted with word frequency in the OFC. We propose that the speed of bottom up processing as manipulated by word frequency modulates OFC predictability responses. Therefore, breaches of expec tation activate the OFC particularly in low frequency words. Finally, the present study shows that the high sampling frequency of NIRS makes it an excellent method for the ecologically valid investigation of the hemodynamic responses in the ultra rapid event succession of words during naturally paced reading.

\section{Occipital predictability effect}

A significant deoxygenation main effect was observed in a channel likely localized in the left middle occipital cortex. The planned compar ison showed that low predictability words led to a greater [deoxy $\mathrm{Hb}$ ]

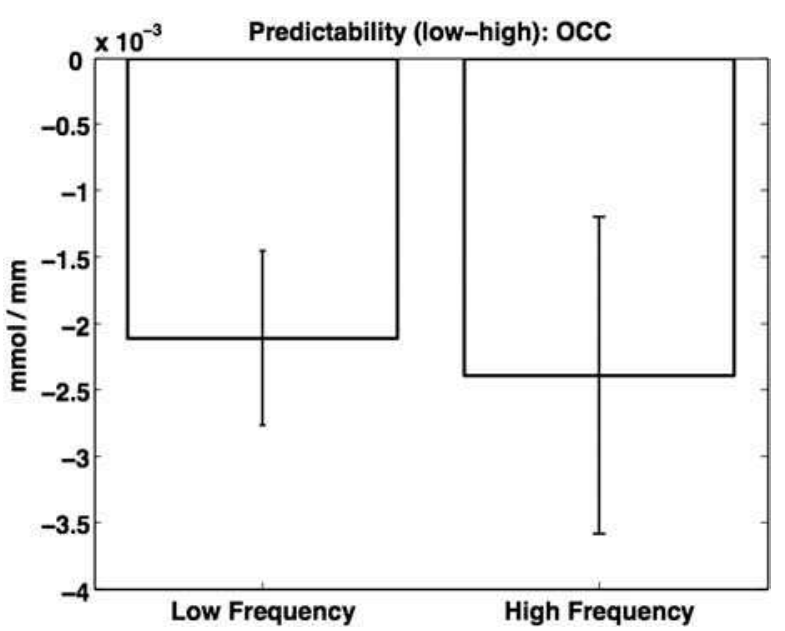

Fig. 3. The beta-value differences index a [deoxy-Hb] decrease for low- relative to highpredictability words. This suggests greater OCC activation to low-predictability words. This decrease is apparent in low- and in high-frequency words. Error bars index (cross-subject) standard errors of this difference. decrease in comparison to high predictability words (cf. Alink et al., 2010).

To examine the empirical hemodynamic responses, we conducted a deconvolution analysis (Koch et al., 2009). Therefore, we subtracted the model based estimates of the non target events from the empirical time course, averaged these time courses relative to the mean of a $1 \mathrm{~s}$ pre stimulus epoch, and generated a low high predictability contrast. Fig. 6 shows that [deoxy $\mathrm{Hb}$ ] decreases due to low predictability as compared to high predictability words in channel OCC. Thus the time course of the hemodynamic response provides converging evidence that low predictability engages greater occipital activation than high predictability in words (Buxton et al., 2004).

These findings confirm a previous suggestion by Dambacher et al. (2009). Using the same experimental design, they found an early predictability effect in an ERP time frame between 50 and $90 \mathrm{~ms}$. A relatively posterior ERP distribution made the authors suggest that this follows from activation in visual cortex (e.g., Rauss et al., 2011). The present results agree with this hypothesis. We suggest that, before stimulus presentation, visual feature templates of expected words are pre activated in the occipital cortex. Therefore, less novel neural energy must be recruited at the time of stimulus presentation to represent these visual features. In contrast, activating the visual features of an unex pected word is driven by poststimulus bottom up processing, entailing greater neural energy demands.

The occipital prediction error effect during reading poses clear constraints on connectionist models of associations between words. The activation of visual feature representations in an interactive activa tion model is associated with neural activation in the occipital cortex (Grainger and Jacobs, 1996; McClelland and Rumelhart, 1981; Schurz et al., 2010; Vinckier et al., 2007). In bottom up processing, visual fea tures activate letters, which in turn activate word representations. These letter and word layers can be associated with more anterior regions in the ventral visual stream (Schurz et al., 2010; Vinckier et al., 2007). The scientific community seems to converge on the opinion, however, that these representations interface to higher order processes such as semantic associations between words (Dehaene and Cohen, 2011; Price and Devlin, 2011). McClelland and Rogers (2003) reviewed evidence on how such associations are generated. To define an established knowledge system of outlearned long term representa tions, an interactive activation model was extended by an associative layer (Hofmann et al., 2011). It is based on a symbolic version of Hebbian learning: Associations between words are defined by the likelihood of words occurring together in the sentences of a large corpus (Quasthoff et al., 2006). Following this rationale, each associated word in a sentence context can be seen as a contextual cue associated with the word expected to occur (Bar et al., 2007). The evidence that predictive associations affect occipital activations suggests an inclusion of top down connections from higher layers down to the lowest levels of visual feature representations (Hofmann et al., 2011). Therefore, the visual features of expected words become ac tive. This provides empirical evidence for the principle of interactiv ity, which suggests that lower level representations are activated by higher levels (McClelland, 1993). For example, because the most strongly associated word to 'composer' is 'music', its visual features are pre activated in the occipital cortex (cf. Fig. 1; Hofmann et al., 2011; 


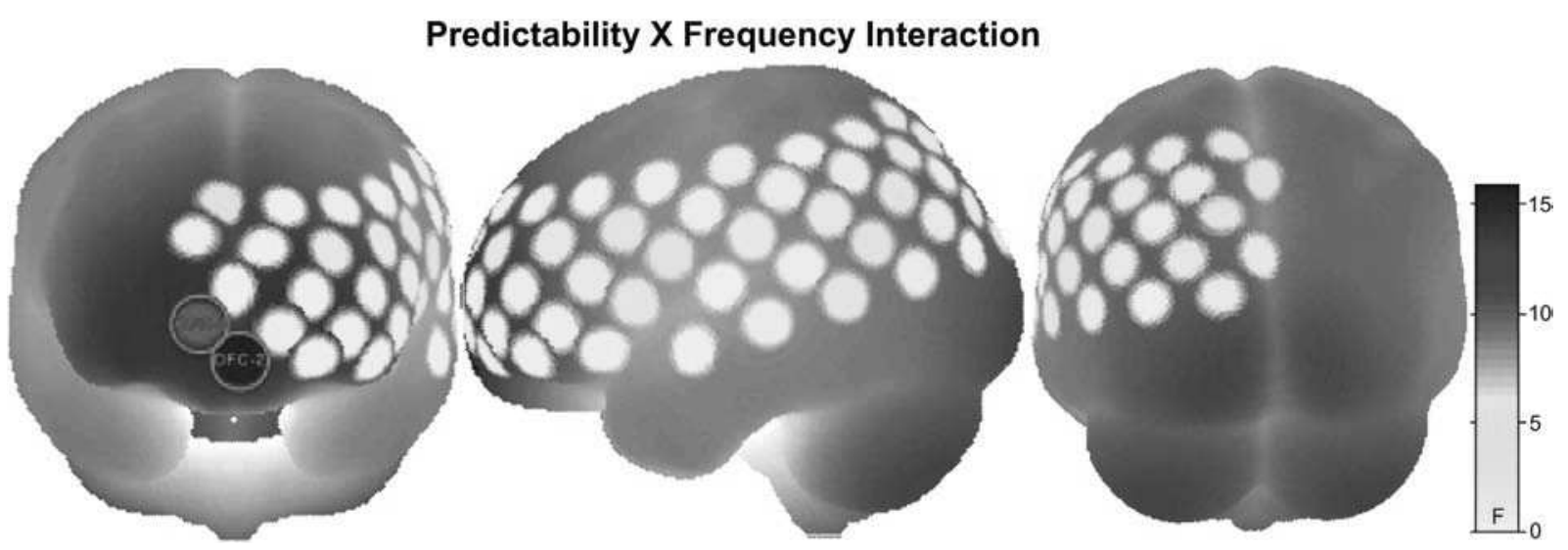

Fig. 4. The figure shows the results of the repeated measures ANOVAs for the interaction of word frequency and predictability. The channels termed OFC-1 and OFC-2 surpassed the Bonferroni-corrected alpha threshold (cf. Fig. 5 and 7).

Quasthoff et al., 2006; http://corpora.uni leipzig.de/). When the critical word is presented, such predictions are validated within $100 \mathrm{~ms}$ after stimulus exposure (Dambacher et al., 2009).

\section{Orbitofrontal interaction of word frequency and predictability}

Previous proposals that OFC responds either to unexpected or to ex pected events were controversial (Bar et al., 2006; Nobre et al., 1999). They did not yet identify a factor that determines whether OFC responds to prediction errors or matches. We observed an interaction of word frequency with predictability in two channels that were most likely localized in the OFC (Tsuzuki et al., 2007).

In channel OFC 1 , the low high predictability contrast was positive for high and negative for low frequency words. This is also reflected in the deconvolution analysis. The left panel of Fig. 7 shows that the low high predictability deoxygenation in OFC 1 increases for high frequency words, while it decreases for low frequency words. The [deoxy $\mathrm{Hb}$ ] increase suggests greater neural activation to high than to low predictability words of high frequency, as compared to low frequency words. In this case, low predictability elicits greater neural activation than high predictability.

In channel OFC 2 , low predictability engaged greater neural activa tion in low frequency words, which is confirmed by a [deoxy $\mathrm{Hb}$ ] decrease of the empirical time course (Fig. 7, right panel). As previous ERP studies proposed that bottom up processing is faster for high than for low frequency words (Dambacher et al., 2006; Hauk and
Pulvermüller, 2004), we suggest that the speed of incoming bottom up information modulates OFC function: In slow bottom up signals, as in low frequency words, greater hemodynamic responses to non predictable words index a failure to predict a target word.

When a word occurs rarely in natural language, its bottom up processing is relatively slow. For example, response times can be more than 100 ms slower for low than for high frequency words (Hofmann et al., 2008). Moreover, low frequency words display a greater ERP amplitude within $200 \mathrm{~ms}$ after stimulus exposure (Dambacher et al., 2006; Hauk and Pulvermüller, 2004). Low frequency words also show greater predictability effects after 300 ms (Dambacher et al., 2006). This suggests that processing of low frequency words was still underway after the stimulus exposure time of $250 \mathrm{~ms}$. Contemporary models of visual word recognition would suggest that phonological processing contributes to the visual recognition of low frequency words in this time frame (Coltheart et al., 2001; Grainger and Holcomb, 2009; Perry et al., 2007; Proverbio et al., 2004). This incomplete processing of low frequency words may elicit OFC activation as a warning signal when the top down prediction does not match the presented stimulus. We propose that such a signal triggers a top down driven search process, initiated by the OFC as a 'monitor' of prediction success (Barbey et al., 2011; Nobre et al., 1999).

As opposed to a [deoxy $\mathrm{Hb}$ ] decrease to low vs. high predictability in low frequency words, OFC 1 revealed an increase in high frequency words (Fig. 5). This interaction indicates greater neural activation to high than to low predictability in high frequency words. In particular,
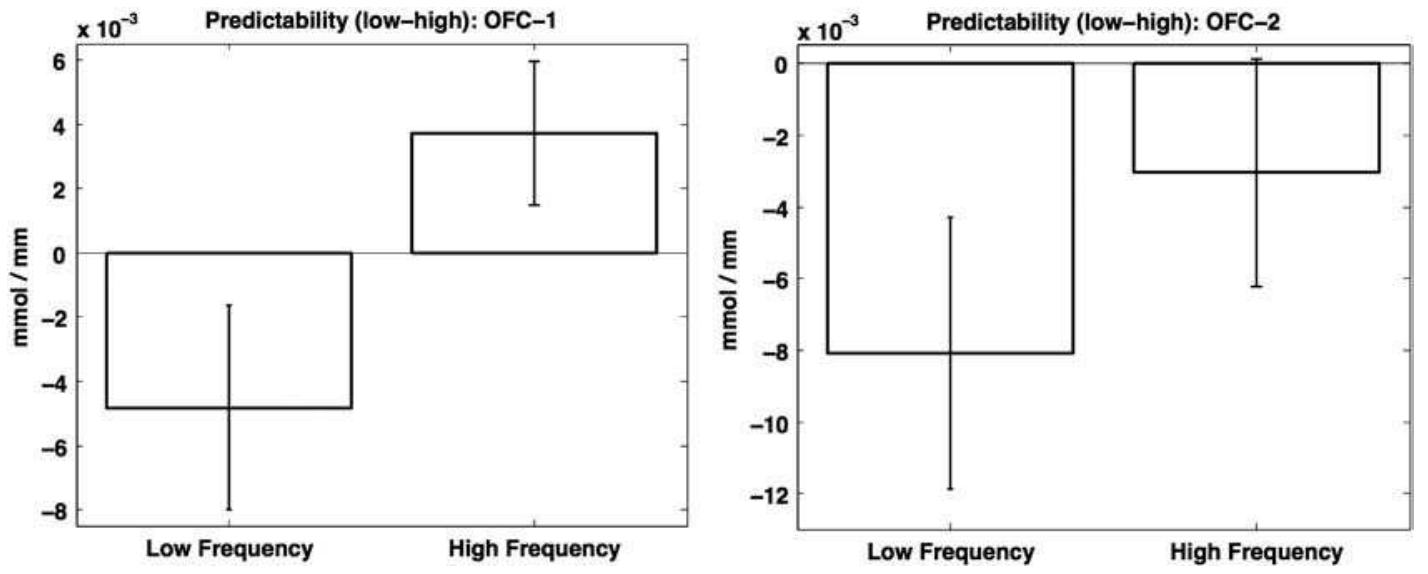

Fig. 5. Beta-value differences for low-high predictability illustrate the Bonferroni-corrected significant interactions. In channel OFC-1, the [deoxy-Hb] difference for low-frequency words became negative, while it became positive for high-frequency words. In channel OFC-2, the deoxygenation decrease was greater for low than for high-frequency words. Error bars indicate the standard errors of the low-high predictability differences across subjects. 


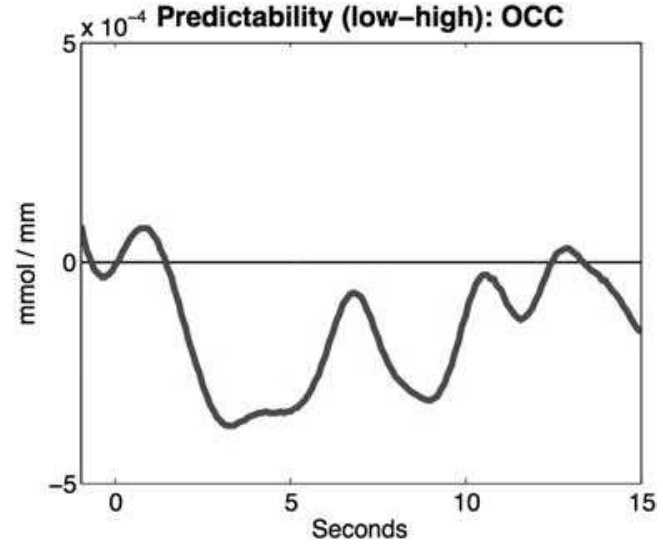

Fig. 6. Empirical time courses of the low-high predictability responses in channel OCC (cf. Fig. 2). The model-based estimates of the non-target events were subtracted in a deconvolution analysis. The figure shows that [deoxy-Hb] to low predictability decreases relative to high predictability. This indexes greater occipital activation for lowpredictability words.

as there is faster bottom up evidence for high compared to low frequency words, associative top down predictions encounter a greater amount of bottom up evidence within the same amount of time (Hofmann et al., 2011; Jacobs et al., 2003; Kveraga et al., 2007; Ullmann, 1995). For words that are both of high frequency and high predictability, OFC activation might reflect a confirmatory 'aha' signal, indexing a match between the predicted and the presented word (Bar et al., 2006).

There are Positron Emission Topography findings that suggest the OFC's role in matching top down predictions in the broader context of language processing. Nathaniel James and Frith (2002) showed that neural activation is greater, when participants complete a cloze test by naming a matching word, as compared to a completion that does not fit into this sentence. Buckner et al. (1995) showed that OFC regions are activated when participants generate a semantically matching verb to a noun stimulus (cf. Price, 2012). Further, Maguire et al. (1999) found a significant correlation between story comprehension and OFC activation, which indicates that the words matched better with prior expectations. Therefore, novel information can be integrated more seamlessly into an existing knowledge structure.

We suggest that complementary roles in matching and violated pre dictions could be reconciled within the theoretical framework of Schoenbaum et al. (2009). They suggest that the OFC's primary function lies in providing a signal that changes associative representations. This occurs by signaling prediction errors that function as a teaching signal for other brain regions (Friston, 2010). While they emphasized the role of the OFC as one of the brain regions that index such prediction errors, the present study provides evidence that this prediction error effect is modulated by the amount of bottom up information during reading, as manipulated by word frequency. If matching predictions en gage the OFC (Bar et al., 2007), existing associative connections between neurons could be strengthened, when the association predicted the future event. This would also support the Bayesian brain hypothesis (Friston, 2010), which proposes that the brain uses internal probabilis tic models to update posterior beliefs, which optimizes future predic tions (Duarte et al., 2009; Kiebel et al., 2008). Thus, OFC lesions to disrupt social behavior might actually result from the failure to antici pate the consequences of the patient's actions, and thus the failure to apply and update such conditional probabilities (Bechara et al., 2000). However, it requires further research to determine whether OFC signals in such a rapid recognition situation as reading lead to an update of the associative connections, i.e. whether OFC activation stimulates learning (Kuchinke et al., 2013). During reading, however, we conclude that the OFC supervises the short term spreading of associative activation in a long term memory structure (Anderson, 1983; Bar et al., 2007; Collins and Loftus, 1975; Hofmann et al., 2011), given a particular amount of available bottom up information. Therefore, OFC allows humans to flexibly adopt to environmental demands.

\section{Fast sampling and ultra rapid event succession during reading}

As recent fMRI studies started to approach the goal of addressing hemodynamic responses during natural reading (Marsman et al., 2011; Richlan et al., 2013), we think that fNIRS and fMRI as methods providing either superior temporal or superior spatial resolution favor ably complement one another (Devor et al., 2012).

The present study took one important step towards addressing the hemodynamic response during natural reading, by demonstrating straightforward occipital predictability effects using rapid serial visual presentation with inter stimulus intervals of less than $300 \mathrm{~ms}$ (Alink et al., 2010; Fischer et al., 2012). While a superior temporal resolution has previously been discussed as a promising potential of the NIRS (Devor et al., 2012), the present study takes full advantage of the rapid sampling frequency.

However, Marsman et al. (2011) suggested that undersampling i.e. a lower sampling than event frequency is not a problem for condition wise analyses (Richlan et al., 2013). This is because samples
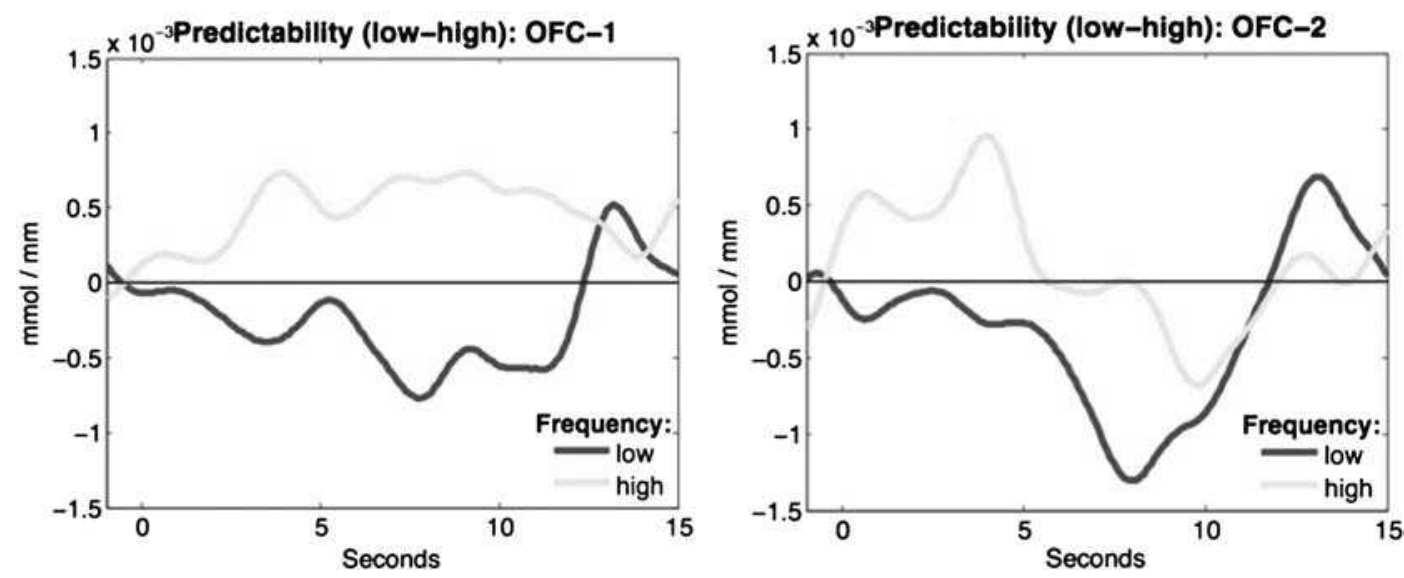

Fig. 7. Empirical time courses of the low-high predictability responses for low- and high-frequency words. The left panel shows that a decrease of [deoxy-Hb] is obtained for low-frequency words (dark blue) in channel OFC-1 (cf. Fig. 4). This suggests greater orbitofrontal activation in low-predictability words of low frequency. In contrast, [deoxy-Hb] increased due to lowpredictability in high-frequency words (light blue): This suggests greater neural activation to high- than to low-predictability words. In the right panel, OFC-2 showed a [deoxy-Hb] decrease due to low predictability in low-frequency words. 
collected at a different latency from event onset do effectively increase the sampling of the condition over actual recording sampling frequency. Undersampling is equally sufficient when several trials in an experi ment contribute to an item level regressor (Yarkoni et al., 2008). The critical level at which undersampling would not work anymore is the single trial level: When six to ten words are perceived within a period of $2 \mathrm{~s}$, a subsequent single data point can hardly inform about which of the events caused the deoxygenation to what degree. Deconvolution at the single trial level will particularly aid multivariate data fusion, when each fixation time is compared to the hemodynamic response it elicited (cf. Ritter and Villringer, 2006, for a similar discussion about EEG and fMRI). These are challenges, however, for future stud ies, which should use either non standard fMRI scanning parameters (Sabatinelli et al., 2009), or a NIRS sampling frequency of up to $300 \mathrm{~Hz}{ }^{1}$

Speed is crucially important, because we know from ERP studies that an unnaturally slow reading pace can dramatically change the strategy with which readers recognize words in sentences (Dambacher et al., 2012). Dambacher et al. (2012) showed that an early ERP interaction between frequency and predictability occurs only at near natural read ing rates. If we assume that the OFC interaction between predictability and frequency reflects the ERP interaction starting previous to $150 \mathrm{~ms}$ after stimulus exposure (Dambacher et al., 2012), we would expect also the OFC interaction to vanish with greater inter stimulus intervals during unnaturally slow word recognition. This hypothesis would be fully compatible with Bar et al.'s (2006) proposal that the predictive function of the OFC is engaged around $130 \mathrm{~ms}$ after stimulus exposure, when stimulus presentation rate is fast enough such that top down pre dictions are required.

Though fNIRS will never reach the spatial resolution of magnetic resonance imaging, this speed adds to the list of advantages that make fNIRS extremely attractive for the ecologically valid study of reading: It is also a silent method that allows undisturbed reading while sitting at a table.

Finally, we propose that a rapid pace circumvents a 'default mode' of brain function (Gusnard and Raichle, 2001). During long inter stimulus intervals, participants display brain activation that is not under experi mental control; rather, brain activation is generated intrinsically. Some have called the cognitive counterpart of the default mode activation 'mindwandering' (Bar et al., 2007), while others propose that this net work directly reflects the semantic processes during verbal thinking (Binder et al., 2009). To our knowledge, there is no language task that successfully defined a resting condition without any language process ing (e.g. Price, 2012; Rossi et al., 2012). Such a baseline could be compared to a language processing condition, as this is possible for motor vs. non motor activation (e.g. Boden et al., 2007). This is not possible, because thoughts are language based in the so called resting periods (Binder et al., 2009), which can lead to relative deactivation in the non resting condition (e.g., Brink et al., 2011; Gusnard and Raichle, 2001). Therefore, it may be better to compare language stimuli to similar non language stimuli (Price, 2012). We suggest that slowly paced word recognition tasks lead to language related cognitive pro cesses that are not under experimental control. In contrast, keeping the participants busy with a rapid event succession may lead to a model that more successfully captures all cognitive processes in an experiment.

In conclusion, our data demonstrate that fNIRS provides sufficient temporal resolution for investigating OFC activity, while this region su pervises associative processes during reading (Anderson, 1983; Bar et al., 2007; Collins and Loftus, 1975; Hofmann et al., 2011). Induced by a suitable context sentence, the expectation of a word pre activates its visual features. Therefore, less occipital neural energy is consumed than during the presentation of an unexpected word, in which visual

\footnotetext{
${ }^{1}$ http://www.shimadzu.com/an/lifescience/imaging/nirs/nirs_top.html.
}

features must be exclusively activated post stimulus. We interpret the interaction between word frequency and predictability in terms of a dynamic interplay between top down and bottom up processes. OFC function is modulated by the amount of available bottom up informa tion: With a low amount of bottom up information in low frequency words, stimulus processing will take time. If additionally top down predictions do not match the stimulus, the OFC response may initiate a search process to attribute meaning to the word. In contrast, when a greater amount of bottom up information is available in high frequency words, the OFC response is less likely to index a prediction error. Future studies will have to evaluate whether a sufficient amount of bottom up information will cause an OFC signal indexing a successful match. In sum, we conclude that it depends on the amount of available bottom up information, whether the OFC responds to breaches of expectation during reading.

\section{Acknowledgments}

We like to thank Dominik Diesing for data acquisition and the Deutsche Forschungsgemeinschaft for the financial support (grants JA 823/4 1, 42 to Arthur Jacobs, and HO5139/2 1 to Markus Hofmann).

\section{References}

Alink, A., Schwiedrzik, C.M., Kohler, A., Singer, W., Muckli, L., 2010. Stimulus predictability reduces responses in primary visual cortex. J. Neurosci. 30, 2960-2966.

Anderson, J.R., 1983. A spreading activation theory of memory. J. Verbal Learn. Verbal Behav. 22, 261-295.

Bar, M., Kassam, K.S., Ghuman, A.S., Boshyan, J., Schmid, A.M., Dale, A.M., Hämäläinen, M.S. , Marinkovic, K., Schacter, D.L., Rosen, B.R., Halgren, E., 2006. Top-down facilitation of visual recognition. Proc. Natl. Acad. Sci. U. S. A. 103, 449-454.

Bar, M., Aminoff, E., Mason, M., Fenske, M., 2007. The units of thought. Hippocampus 6, $420-428$.

Barbey, A.K., Koenigs, M., Grafman, J., 2011. Orbitofrontal contributions to human working memory. Cereb. Cortex 21, 789-795.

Bechara, A., Damasio, H., Damasio, A.R., 2000. Emotion, decision making and the orbitofrontal cortex. Cereb. Cortex 10, 295-307.

Berns, G.S., McClure, S.M., Pagnoni, G., Montague, P.R., 2001. Predictability modulates human brain response to reward. J. Neurosci. 21, 2793-2798.

Binder, J.R., Desai, R.H., Graves, W.W., Conant, L.L., 2009. Where is the semantic system? A critical review and meta-analysis of 120 functional neuroimaging studies. Cereb. Cortex 19, 2767-2796.

Boden, S., Obrig, H., Köhncke, C., Benav, H., Koch, S.P., Steinbrink, 2007. The oxygenation response to functional stimulation: is there a physiological meaning to the lag between parameters? NeuroImage 36, 100-107.

Bohrn, I.C., Altmann, U., Lubrich, O., Menninghaus, W., Jacobs, A.M., 2012. Old proverbs in new skins - an FMRI study on defamiliarization. Front. Psychol. 3, 204.

Boynton, G.M., Engel, S.A., Glover, G.H., Heeger, D.J., 1996. Linear systems analysis of functional magnetic resonance imaging in human V1. J. Neurosci. 16, 4207-4221.

Brink, T.T., Urton, K., Held, D., Kirilina, E., Hofmann, M.J., Klann-Delius, G., Jacobs, A.M., Kuchinke, L., 2011. The role of orbitofrontal cortex in processing empathy stories in 4- to 8-year-old children. Front. Psychol. 2, 80.

Buckner, R.L., Raichle, M.E., Petersen, S.E., 1995. Dissociation of human prefrontal cortical areas across different speech production tasks and gender groups. J. Neurophysiol. 74, 2163-2173.

Bullmore, E. Brammer, M. Williams, S.C.R., Rabe-Hesketh, S, Janot, N., David, A., Mellers, J. Howard, R., Sham, P., 1996. Statistical methods of estimation and inference for functional MR image analysis. Magn. Reson. Med. 35, 261-277.

Burock, M.A., Buckner, R.L., Woldorff, M.G., Rosen, B.R., Dale, A.M., 1998. Randomized event-related experimental designs allow for extremely rapid presentation rates using functional MRI. Neuroreport 9, 3735-3739.

Buxton, R.B., Uludag, K., Dubowitz, D.J., Liu, T.T., 2004. Modeling the hemodynamic response to brain activation. Neurolmage 23, S220-S233.

Chen, H.-C., Vaid, J., Boas, D.A., Bortfeld, H., 2011. Examining the phonological neighborhood density effect using near infrared spectroscopy. Hum. Brain Mapp. 32, 1363-1370.

Cochrane, D., Orcutt, G.H., 1949. Application of least squares regression to relationships containing auto-correlated error terms. J. Am. Stat. Assoc. 44, 32-61.

Collins, A.M., Loftus, E.F., 1975. A spreading-activation theory of semantic processing. Psychol. Rev. 82, 407-428.

Coltheart, M., Rastle, K., Perry, C., Langdon, R., Ziegler, J., 2001. DRC: a dual route cascaded model of visual word recognition and reading aloud. Psychol. Rev. 108, 204-256.

Cope, M., Delpy, D.T., 1988. System for long-term measurement of cerebral blood and tissue oxygenation on newborn infants by near infra-red transillumination. Med. Biol. Eng. Comput. 26, 289-294.

Dambacher, M., Kliegl, R., Hofmann, M., Jacobs, A.M., 2006. Frequency and predictability effects on event-related potentials during reading. Brain Res. 1084, 89-103.

Dambacher, M., Rolfs, M., Goellner, K., Kliegl, R., Jacobs, A.M., 2009. Event-related potentials reveal rapid verification of predicted visual input. PLoS One 4, e5047. 
Dambacher, M., Dimigen, O., Braun, M., Wille, K., Jacobs, A.M., Kliegl, R., 2012. Stimulus onset asynchrony and the timeline of word recognition: event-related potentials during sentence reading. Neuropsychologia 50, 1852-1870.

Dehaene, S., Cohen, L., 2011. The unique role of the visual word form area in reading. Trends Cogn. Sci. 15, 254-262.

Devor, A., Sakadzic, S., Srinivasan, V.J., Yaseen, M.A., Nizar, K., Saisan, P.A., Tian, P., Dale, A.M., Vinogradov, S.A., Franceschini, M.A., Boas, D.A., 2012. Frontiers in optical imaging of cerebral blood flow and metabolism. J. Cereb. Blood Flow Metab. 32, 1259-1276.

Duarte, A., Henson, R.N., Knight, R.T., Emery, T., Graham, K.S., 2009. Orbito-frontal cortex is necessary for temporal context memory. J. Cogn. Neurosci. 22, 1819-1831.

Fiebach, C.J., Vos, S.H., Friederici, A.D., 2004. Neural correlates of syntactic ambiguity in sentence comprehension for low and high span readers. J. Cogn. Neurosci. 16, 1562-1575

Fischer, R., Plessow, F., Ruge, H., 2012. Priming of visual cortex by temporal attention? The effects of temporal predictability on stimulus(-specific) processing in early visual cortical areas. Neurolmage 66C, 261-269.

Friston, K., 2010. The free-energy principle: a unified brain theory? Nat. Rev. Neurosci. 11, $127-138$

Geyken, A., 2007. The DWDS corpus: a reference corpus for the German language of the 20th century. In: Fellbaum, C. (Ed.), Idioms and Collocations: Corpus-based Linguistic, Lexicographic Studies. Continuum Press, London, pp. 23-40.

Grainger, J., Holcomb, P.J., 2009. Watching the word go by: on the time-course of component processes in visual word recognition. Lang. Ling. Compass 3 , $128-156$.

Grainger, J., Jacobs, A.M., 1996. Orthographic processing in visual word recognition: a multiple read-out model. Psychol. Rev. 103, 518-565.

Gusnard, D.A., Raichle, M.E., 2001. Searching for a baseline: functional imaging and the resting human brain. Nat. Rev. Neurosci. 2, 685-694.

Haeussinger, F.B., Heinzel, S., Hahn, T., Schecklmann, M., Ehlis, A.-C., Fallgatter, A.J., 2011. Simulation of near-infrared light absorption considering individual head and prefrontal cortex anatomy: implications for optical neuroimaging. PLoS One 6, e26377.

Hauk, O., Pulvermüller, F., 2004. Effects of word length and frequency on the human event-related potential. Clin. Neurophysiol. 115, 1090-1103.

Hebb, D., 1949. The Organization of Behavior. Wiley, New York

Hofmann, M.J., Herrmann, M.J., Dan, I., Obrig, H., Conrad, M., Kuchinke, L., Jacobs, A.M., Fallgatter, A.J., 2008. Differential activation of frontal and parietal regions during visual word recognition: an optical topography study. NeuroImage 40 , 1340-1349.

Hofmann, M.J., Kuchinke, L., Biemann, C., Tamm, S., Jacobs, A.M., 2011. Remembering words in context as predicted by an associative read-out model. Front. Psychol. 2, 252

Jacobs, A.M., Graf, R., Kinder, A., 2003. Receiver operating characteristics in the lexical decision task: evidence for a simple signal-detection process simulated by the multiple read-out model. J. Exp. Psychol. Learn. Mem. Cogn. 29, 481-488.

Kiebel, S.J., Daunizeau, J., Friston, K.J., 2008. A hierarchy of time-scales and the brain. PLoS Comput. Biol. 4, e1000209.

Kirilina, E., Jelzow, A., Heine, A., Niessing, M., Wabnitz, H., Brühl, R., Ittermann, B., Jacobs, A.M., Tachtsidis, I., 2012. The physiological origin of task-evoked systemic artefacts in functional near infrared spectroscopy. Neurolmage 61, 70-81.

Kliegl, R., Nuthmann, A., Engbert, R., 2006. Tracking the mind during reading: the influence of past, present, and future words on fixation durations. J. Exp. Psychol. Gen. $135,12-35$

Koch, S.P., Werner, P., Steinbrink, J., Fries, P., Obrig, H., 2009. Stimulus-induced and statedependent sustained gamma activity is tightly coupled to the hemodynamic response in humans. J. Neurosci. 29, 13962-13970.

Kringelbach, M.L., Rolls, E.T., 2004. The functional neuroanatomy of the human orbitofrontal cortex: evidence from neuroimaging and neuropsychology. Prog. Neurobiol. 72, 341-372.

Kuchinke, L., Fritzemeier, S., Hofmann, M.J., Jacobs, A.M., 2013. Neural correlates of episodic memory: associative memory and confidence drive hippocampus activations. Behav. Brain Res. 254, 92-101.

Kutas, M., Federmeier, K.D., 2011. Thirty years and counting: finding meaning in the N400 component of the event-related brain potential (ERP). Annu. Rev. Psychol. 62, 621-647.

Kveraga, K., Ghuman, A.S., Bar, M., 2007. Top-down predictions in the cognitive brain. Brain Cogn. 65, 145-168

Maguire, E.A., Frith, C.D., Morris, R.G., 1999. The functional neuroanatomy of comprehension and memory: the importance of prior knowledge. Brain 122, 1839-1850.

Mandeville, JB., Marota, J.J. Ayata, C., Moskowitz, M.A. Weisskoff, R.M., Rosen, B.R. 1999. Evidence of a cerebrovascular postarteriole windkessel with delayed compliance. J. Cereb. Blood Flow Metab. 5, 679-689.

Marsman, J.B.C., Renken, R., Velichkovsky, B.M., Hooymans, J.M.M., Cornelissen, F.W., 2011. Fixation based event-related fmri analysis: using eye fixations as events in functional magnetic resonance imaging to reveal cortical processing during the free exploration of visual images. Hum. Brain Mapp. 33, 307-318.

McClelland, J.L., 1993. Toward a theory of information processing in graded, random, and interactive networks. In: Meyer, D.E., Kornblum, S. (Eds.), Attention and Performance XIV: Synergies in Experimental Psychology, Artificial Intelligence, and Cognitive Neuroscience. MIT Press, Cambridge, pp. 655-688.

McClelland, J.L., Rogers, T.T., 2003. The parallel distributed processing approach to semantic cognition. Nat. Rev. Neurosci. 4, 310-322.

McClelland, J.L., Rumelhart, D.E., 1981. An interactive activation model of context effects in letter perception: part 1. An account of basic findings. Psychol. Rev. 5, 375-407.
Nathaniel-James, D., Frith, C., 2002. The role of the dorsolateral prefrontal cortex: evidence from the effects of contextual constraint in a sentence completion task. Neurolmage $16,1094-1102$.

Nobre, A.C., Coull, J.T., Frith, C.D., Mesulam, M.M., 1999. Orbitofrontal cortex is activated during breaches of expectation in tasks of visual attention. Nat. Neurosci. 2, 11-12.

Noguchi, Y., Takeuchi, T., Sakai, K.L., 2002. Lateralized activation in the inferior frontal cortex during syntactic processing: event-related optical topography study. Hum. Brain Mapp. 17, 89-99.

O'Doherty, J.P., Dayan, P., Friston, K., Critchley, H., Dolan, R.J., 2003. Temporal difference models and reward-related learning in the human brain. Neuron 38 329-337.

Obleser, J., Kotz, S.A., 2010. Expectancy constraints in degraded speech modulate the language comprehension network. Cereb. Cortex 20, 633-640.

Perry, C., Ziegler, J.C., Zorzi, M., 2007. Nested incremental modeling in the development of computational theories: the CDP + model of reading aloud. Psychol. Rev. 114 273-315.

Petrides, M., 2007. The orbitofrontal cortex: novelty, deviation from expectation, and memory. Ann. N. Y. Acad. Sci. 1121, 33-53.

Plichta, M.M., Herrmann, M.J., Baehne, C.G., Ehlis, A.-C., Richter, M.M., Pauli, P., Fallgatter A.J., 2006. Event-related functional near-infrared spectroscopy (fNIRS): are the measurements reliable? NeuroImage 31, 116-124

Plichta, M.M., Heinzel, S., Ehlis, A.-C., Pauli, P., Fallgatter, A.J., 2007. Model-based analysis of rapid event-related functional near-infrared spectroscopy (NIRS) data: a parametric validation study. NeuroImage 35, 625-634.

Price, C.J., 2012. A review and synthesis of the first 20 years of PET and fMRI studies of heard speech, spoken language and reading. Neurolmage 62, 816-847.

Price, C.J., Devlin, J.T., 2011. The interactive account of ventral occipitotemporal contributions to reading. Trends Cogn. Sci. 15, 246-253.

Proverbio, A.M., Vecchi, L., Zani, A., 2004. From orthography to phonetics: ERP measures of grapheme-to-phoneme conversion mechanisms in reading. J. Cogn. Neurosci. 16 301-317.

Pulvermüller, F., 1999. Words in the brain's language. Behav. Brain Sci. 22, 253-336.

Quasthoff, U., Richter, M., Biemann, C., 2006. Proceedings of LREC-06 (Genova), pp. $10-13$.

Raichle, M.E., Mintun, M.A., 2006. Brain work and brain imaging. Annu. Rev. Neurosci. 29, 449-476.

Rao, R.P., Ballard, D.H., 1999. Predictive coding in the visual cortex: a functional interpretation of some extra-classical receptive-field effects. Nat. Neurosci. 2 $79-87$

Rauss, K., Schwartz, S., Pourtois, G., 2011. Top-down effects on early visual processing in humans: a predictive coding framework. Neurosci. Biobehav. Rev. 35, 1237-1253.

Rayner, K., 1998. Eye movements in reading and information processing: 20 years of research. Psychol. Bull. 124, 372-422.

Richlan, F., Gagl, B., Hawelka, S., Braun, M., Schurz, M., Kronbichler, M., Hutzler, F., 2013. Fixation-related fMRI analysis in the domain of reading research: using self-paced eye movements as markers for hemodynamic brain responses during visual letter string processing. Cereb. Cortex. http://dx.doi.org/10.1093/cercor/ bht117.

Ritter, P., Villringer, A., 2006. Simultaneous EEG-fMRI. Neurosci. Biobehav. Rev. 30, 823-838.

Rossi, S., Telkemeyer, S., Wartenburger, I., Obrig, H., 2012. Shedding light on words and sentences: near-infrared spectroscopy in language research. Brain Lang. 121, 152-163.

Sabatinelli, D., Lang, P.J., Bradley, M.M., Costa, V.D., Keil, A., 2009. The timing of emotional discrimination in human amygdala and ventral visual cortex. J. Neurosci. 29 14864-14868.

Sankoh, A.J., Huque, M.F., Dubey, S.D., 1997. Some comments on frequently used multiple endpoint adjustment methods in clinical trials. Stat. Med. 16, 2529-2542.

Schmidt, C.F., Degonda, N., Luechinger, R., Henke, K., Boesiger, P., 2005. Sensitivityencoded (SENSE) echo planar fMRI at $3 \mathrm{~T}$ in the medial temporal lobe. Neurolmage $25,625-641$.

Schoenbaum, G., Roesch, M.R., Stalnaker, T.A., Takahashi, Y.K., 2009. A new perspective on the role of the orbitofrontal cortex in adaptive behaviour. Nat. Rev. Neurosci. 10, 885-892.

Schroeter, M.L., Buecheler, M.M., Mueller, K., Uludag, K., Obrig, H., Lohmann, G. Tittgemeyer, M. Villringer, A von Cramon, Y., 2004. Towards a standard analysis for functional near-infrared imaging. NeuroImage 21, 283-290.

Schurz, M., Sturm, D., Richlan, F., Kronbichler, M., Ladurner, G., Wimmer, H., 2010. A dualroute perspective on brain activation in response to visual words: evidence for a length by lexicality interaction in the visual word form area (VWFA). Neurolmage 49, 2649-2661.

Seidenberg, M.S., McClelland, J.L., 1989. A distributed, developmental model of word recognition and naming. Psychol. Rev. 96, 523-568.

Sereno, S.C., Rayner, K., Posner, M.I., 1998. Establishing a time line of word recognition: evidence from eye movements and event-related potentials. Neuroreport $2195-2200$

Steinbrink, J., Villringer, A., Kempf, F., Haux, D., Boden, S., Obrig, H., 2006. Illuminating the BOLD signal: combined fMRI-fNIRS studies. Magn. Reson. Imaging 24 495-505.

Summerfield, C., Egner, T., 2009. Expectation (and attention) in visual cognition. Trends Cogn. Sci. 13, 403-409.

Tsuzuki, D., Jurcak, V., Singh, A.K., Okamoto, M., Watanabe, E., Dan, I., 2007. Virtual spatial registration of stand-alone fNIRS data to MNI space. Neurolmage 34, 1506-1518.

Tzourio-Mazoyer, N., Landeau, B., Papathanassiou, D., Crivello, F., Etard, O., Delcroix, N. Mazoyer, B., Joliot, M., 2002. Automated anatomical labeling of activations in SPM 
using a macroscopic anatomical parcellation of the MNI MRI single-subject brain. NeuroImage 15, 273-289.

Ullmann, S., 1995. Sequence seeking and counter streams: a computational model fo bidirectional information flow in the visual cortex. Cereb. Cortex 5, 1-11.

Vinckier, F., Dehaene, S., Jobert, A., Dubus, J.P., Sigman, M., Cohen, L., 2007. Hierarchical coding of letter strings in the ventral stream: dissecting the inner organization of the visual word-form system. Neuron 55, 143-156.
Wallis, J.D., 2007. Orbitofrontal cortex and its contribution to decision-making. Annu. Rev. Neurosci. 30, 31-56.

Yarkoni, T., Speer, N.K., Balota, D.A., McAvoy, M.P., Zacks, J.M., 2008. Pictures of a thousand words: investigating the neural mechanisms of reading with extremely rapid eventrelated fMRI. Neurolmage 42, 973-987. 